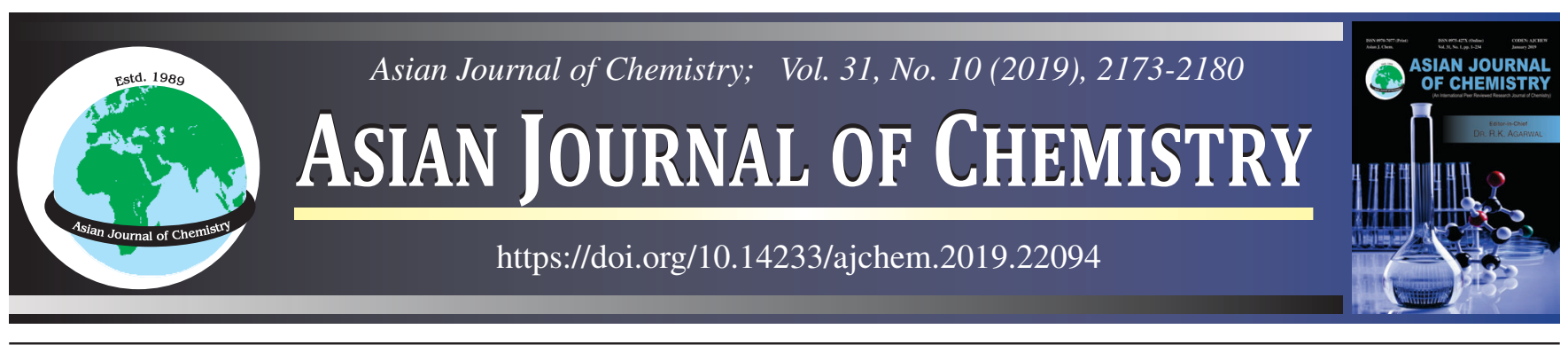

\title{
Partial Molar Volumes and Spectral Studies on Binary Mixtures of $p$-Chloroacetophenone with Aniline and $N$-Alkyl Anilines
}

\author{
M. Radha Sirija, D. Bala and D. Ramachandran*
}

Physico-Chemical Laboratory, Department of Chemistry, Acharya Nagarjuna University, Nagarjuna Nagar-522510, India

*Corresponding author: Email: dittakavirc@ gmail.com

Received: 23 March 2019;

Accepted: 20 April 2019;

Published online: 30 August 2019;

AJC-19518

Ultrasonic velocities $(\mathrm{u})$, viscosities $(\eta)$ and densities $(\rho)$ were measured for the binary mixtures of $p$-chloroacetophenone with aniline, $N$-methylaniline and $N, N$-dimethylaniline over the whole range of composition at different temperatures $(303.15 \mathrm{~K}$ to $318.15 \mathrm{~K})$ and at atmospheric pressure $0.1 \mathrm{MPa}$. Excess molar volume $\left(\mathrm{V}^{\mathrm{E}}\right)$, deviation in viscosity $(\Delta \eta)$ and deviation in isentropic compressibility $\left(\Delta \mathrm{k}_{\mathrm{s}}\right)$ have been calculated and fitted to Redlich-Kister polynomial equation to obtain their coefficients and standard deviations. The analyzed | viscosity data of binary liquid mixtures were used to test the Grunberg-Nissan, Katti-Chaudari and Hind semi-empirical equations. The FTIR spectral study supports the experimental data to explain the molecular interactions between unlike molecules. Partial molar volumes of binary mixtures were evaluated for better understanding of intermolecular interactions of the above binary mixtures.

| Keywords: Excess molar volume, Partial molar volume, Redlich-Kister polynomial equation, Ultrasonic velocities.

ᄂ _ - - - - - - - - - - - - - - - - - - - - - - - - - - -

\section{INTRODUCTION}

Thermodynamic and ultrasonic nature of binary mixtures has great importance. To know about the structural, physicochemical behaviour, intramolecular and intermolecular nature, need of interpretation and analysis of excess parameters and along with liquid mixture theories is necessary. From the values of density $(\rho)$, viscosity $(\eta)$ and ultrasonic velocity (u) and the spectral investigation of Infra-red studies, the behaviour of intermolecular interactions between mixing molecules can be studies. The analysis of thermodynamic properties also shows the degree of deviation from ideal behaviour $[1,2]$.

Previous investigations have been carried out on $p$-chloroacetophenone [3-5] and anilines [6-9] and amines [10,11] have been reported. Literature survey shown that no work has been executed on $p$-chloroacetophenone and aniline, $N$-methylaniline and $N, N$-dimethylaniline at 303.15 to $318.15 \mathrm{~K}$. The effect of molecular size, chain length, shape and extent of molecular association of aromatic and aliphatic amines on volumetric, viscometric and acoustic properties of binary liquid mixtures containing hydrocarbons, esters and alcohols have been studied earlier [12-18].
-Chloroacetophenone as main component with aniline, $\mathrm{N}$-methyl aniline and $\mathrm{N}, \mathrm{N}$-dimethyl aniline are selected for the present investigation of the work. The selected chemicals have wide range of useful applications. $p$-Chloroacetophenone (PCAP) is a molecule having a wide range of application in the manufacture of cosmetics, drugs and perfumes, respectively. Aromatic anilines are used in rubber processing chemicals, herbicides, and dyes \& pigments. A partial molar property is a thermodynamic quantity which indicates how an extensive property of a solution or mixture varies with changes in the molar composition of the mixture at constant temperature and pressure. Partial molar volumes of above mixtures have been calculated at four different temperatures.

\section{EXPERIMENTAL}

$p$-Chloroacetophenone, aniline, $N$-methylaniline, $N, N$ dimethylaniline are used in the present work were purchased from S.D. Fine Chemicals Ltd., India. All the chemicals selected were purified by the method described in the literature [41] and represented in Table-1. Experimental and literature values of density, viscosity and speed of sound of pure liquids viz., $p$ -

This is an open access journal, and articles are distributed under the terms of the Attribution 4.0 International (CC BY 4.0) License. This license lets others distribute, remix, tweak, and build upon your work, even commercially, as long as they credit the author for the original creation. You must give appropriate credit, provide a link to the license, and indicate if changes were made. 


\begin{tabular}{lclcc}
\hline \multicolumn{5}{c}{ TABLE-1 } \\
MATERIALS DESCRIPTION \\
\hline \multicolumn{1}{c}{ Component } & $\begin{array}{c}\text { CAS } \\
\text { number }\end{array}$ & Source & $\begin{array}{c}\text { Mass } \\
\text { fraction } \\
\text { purity }\end{array}$ & $\begin{array}{c}\text { Water } \\
\text { content } \\
(\%)\end{array}$ \\
\hline$p$-Chloroacetophenone & $99-91-2$ & SD-fine & 0.980 & 0.042 \\
Aniline & $71-23-8$ & SD-fine & 0.990 & 0.034 \\
$N$-Methylaniline & $71-36-3$ & SD-fine & 0.990 & 0.033 \\
$N, N$-Dimethylaniline & $71-41-0$ & SD-fine & 0.990 & 0.041 \\
\hline
\end{tabular}

chloroacetophenone, aniline (AN), N-methylaniline (NMA), $\mathrm{N}, \mathrm{N}$-dimethylaniline (NNDMA) at four different temperatures $(303.15,308.15,313.15,318.15 \mathrm{~K})$ are listed in Table-2.

Densities and velocities of pure liquids and mixtures were measured with an Anton-Paar Model DSA-5000M with accuracy in measurements of $\pm 0.0001 \mathrm{~g} / \mathrm{cm}^{3}$. A thermostatically controlled water bath was used. Viscosity measurements at various temperatures were calculated using Ubbelhode viscometer.

TABLE-2

COMPARISON OF EXPERIMENTAL VALUES OF DENSITIES, ULTRASONIC VELOCITY AND VISCOSITIES OF PURE

COMPONENTS WITH THE CORRESPONDING LITERATURE VALUES AT TEMPERATURES (303.15, 308.15, 313.5 AND 318.15) K

\begin{tabular}{|c|c|c|c|c|c|c|c|}
\hline \multirow{2}{*}{ Compound } & \multirow{2}{*}{$\mathrm{T}(\mathrm{K})$} & \multicolumn{2}{|c|}{ Density $(\rho)\left(\mathrm{g} \mathrm{cm}^{-3}\right)$} & \multicolumn{2}{|c|}{ Ultrasonic speed $(\mathrm{u})\left(\mathrm{m} \mathrm{s}^{-1}\right)$} & \multicolumn{2}{|c|}{ Viscosity $(\eta)(\mathrm{mPa} s)$} \\
\hline & & Experimental & Literature & Experimental & Literature & Experimental & Literature \\
\hline \multirow{7}{*}{$p$-Chloroacetophenone } & 303.15 & 1.1859 & $1.1856^{\mathrm{a}}$ & 1412.80 & $1412.00^{\mathrm{a}}$ & 2.3538 & $2.353^{\mathrm{a}}$ \\
\hline & 308.15 & 1.1806 & $1.1812^{\mathrm{a}}$ & 1389.64 & $1389.00^{\mathrm{a}}$ & 2.2924 & $2.292^{\mathrm{a}}$ \\
\hline & & & $1.1813^{\mathrm{b}}$ & & $1390.00^{\mathrm{b}}$ & & $2.293^{\mathrm{c}}$ \\
\hline & & & & & $1395.00^{\mathrm{b}}$ & & \\
\hline & & & & & $1389.98^{\mathrm{d}}$ & & \\
\hline & 313.15 & 1.1755 & & 1366.12 & & 2.2314 & \\
\hline & 318.15 & 1.1704 & & 1343.04 & & 2.1704 & \\
\hline \multirow{28}{*}{ Aniline } & 303.15 & 1.0150 & $1.01330^{\mathrm{e}}$ & 1625.40 & $1623.40^{f}$ & 2.9890 & $3.097^{\mathrm{e}}$ \\
\hline & & & $1.01305^{\mathrm{g}}$ & & $1615.18^{t}$ & & $3.027^{\mathrm{i}}$ \\
\hline & & & $1.01284^{\mathrm{h}}$ & & $1619.20^{\mathrm{g}}$ & & $3.250^{\mathrm{n}}$ \\
\hline & & & $1.01280^{\mathrm{k}}$ & & $1614.50^{\mathrm{i}}$ & & \\
\hline & & & $1.01350^{\mathrm{m}}$ & & $1615.20^{\mathrm{k}}$ & & \\
\hline & & & $1.01290^{\mathrm{m}}$ & & $1615.20^{\mathrm{m}}$ & & \\
\hline & & & $1.01300^{\mathrm{n}}$ & & $1619.30^{\circ}$ & & \\
\hline & & & $1.01290^{\mathrm{n}}$ & & $1614.50^{\circ}$ & & \\
\hline & & & $1.01280^{\circ}$ & & $1614.00^{\mathrm{s}}$ & & \\
\hline & & & $1.01390^{\mathrm{p}}$ & & $1615.00^{\mathrm{s}}$ & & \\
\hline & & & $1.01310^{\mathrm{p}}$ & & & & \\
\hline & & & $1.01318^{\mathrm{s}}$ & & & & \\
\hline & & & $1.01317^{\mathrm{s}}$ & & & & \\
\hline & 308.15 & 1.0106 & $1.00890^{\mathrm{e}}$ & 1605.00 & $1601.88^{t}$ & 2.6430 & $2.663^{\mathrm{e}}$ \\
\hline & & & $1.00870^{\mathrm{n}}$ & & $1602.95^{\mathrm{r}}$ & & $2.800^{\mathrm{n}}$ \\
\hline & & & $1.00860^{\mathrm{n}}$ & & & & $2.635^{\mathrm{i}}$ \\
\hline & & & $1.00867^{\mathrm{r}}$ & & & & \\
\hline & & & $1.00890^{\mathrm{t}}$ & & & & \\
\hline & 313.15 & 1.0062 & $1.00460^{\mathrm{e}}$ & 1585.30 & $1588.29^{t}$ & 2.2970 & $2.329^{\mathrm{e}}$ \\
\hline & & & $1.00490^{\mathrm{k}}$ & & $1582.6^{\mathrm{m}}$ & & $2.420^{\mathrm{n}}$ \\
\hline & & & $1.00436^{\mathrm{m}}$ & & $1580.05^{\mathrm{m}}$ & & $2.304^{\mathrm{i}}$ \\
\hline & & & $1.00463^{\mathrm{m}}$ & & & & \\
\hline & & & $1.00450^{\mathrm{m}}$ & & & & \\
\hline & & & $1.00490^{\mathrm{m}}$ & & & & \\
\hline & & & $1.00440^{\mathrm{n}}$ & & & & \\
\hline & & & $1.00420^{\mathrm{n}}$ & & & & \\
\hline & 318.15 & 1.0018 & $1.00020^{\mathrm{e}}$ & 1565.4 & $1574.41^{\mathrm{t}}$ & 1.951 & $2.057^{\mathrm{e}}$ \\
\hline & & & $1.00090^{\mathrm{t}}$ & & & & $2.047^{\mathrm{i}}$ \\
\hline \multirow{17}{*}{$N$-Methylaniline } & 303.15 & 0.9806 & $0.97770^{\mathrm{e}}$ & 1538.9 & $1545.50^{\mathrm{g}}$ & 1.722 & $1.734^{\mathrm{e}}$ \\
\hline & & & $0.97801^{\mathrm{g}}$ & & $1551.00^{\mathrm{j}}$ & & $1.965^{\mathrm{j}}$ \\
\hline & & & $0.98172^{\mathrm{j}}$ & & $1548.30^{\mathrm{k}}$ & & $1.963^{\mathrm{e}}$ \\
\hline & & & $0.98170^{\mathrm{e}}$ & & $1546.00^{\circ}$ & & \\
\hline & & & $0.97820^{\mathrm{k}}$ & & $1546.90^{r}$ & & \\
\hline & & & $0.97828^{\circ}$ & & $1545.61^{t}$ & & \\
\hline & & & $0.97823 r$ & & & & \\
\hline & & & $0.97830^{t}$ & & & & \\
\hline & 308.15 & 0.9765 & $0.97360^{\mathrm{e}}$ & 1518 & $1528.59^{t}$ & 1.567 & $1.547^{\mathrm{e}}$ \\
\hline & & & $0.97424^{r}$ & & $1528.15^{\mathrm{r}}$ & & \\
\hline & & & $0.97400^{t}$ & & & & \\
\hline & 313.15 & 0.9724 & $0.96960^{\mathrm{e}}$ & 1498.5 & $1511.63^{t}$ & 1.412 & $1.402^{\mathrm{e}}$ \\
\hline & & & $0.96980^{\mathrm{k}}$ & & $1512.40^{\mathrm{k}}$ & & \\
\hline & & & $0.96990^{\mathrm{k}}$ & & & & \\
\hline & & & $0.96960^{\mathrm{k}}$ & & & & \\
\hline & 318.15 & 0.9683 & $0.96580^{\mathrm{e}}$ & 1478.02 & $1494.38^{t}$ & 1.257 & $1.269^{\mathrm{e}}$ \\
\hline & & & $0.96520^{\mathrm{t}}$ & & & & \\
\hline
\end{tabular}




\begin{tabular}{|c|c|c|c|c|c|c|c|}
\hline \multirow{20}{*}{$N, N$-Dimethylaniline } & 303.15 & 0.9481 & $0.94800^{\mathrm{e}}$ & 1462.8 & $1479.00^{\mathrm{a}}$ & 1.176 & $1.180^{\mathrm{e}}$ \\
\hline & & & $0.94770^{\mathrm{n}}$ & & $1468.00^{\mathrm{q}}$ & & $1.174^{\mathrm{u}}$ \\
\hline & & & $0.94800^{\mathrm{n}}$ & & $1468.00^{t}$ & & $1.172^{\mathrm{v}}$ \\
\hline & & & $0.94767^{\mathrm{n}}$ & & & & $1.180^{\mathrm{n}}$ \\
\hline & & & $0.94840^{\mathrm{q}}$ & & & & \\
\hline & & & $0.94800^{\mathrm{q}}$ & & & & \\
\hline & & & $0.94833^{q}$ & & & & \\
\hline & & & $0.94790^{t}$ & & & & \\
\hline & 308.15 & 0.9435 & $0.94390^{\mathrm{e}}$ & 1442.3 & $1462^{c}$ & 1.106 & $1.096^{\mathrm{e}}$ \\
\hline & & & $0.95173^{\mathrm{a}}$ & & $1449.74^{\mathrm{t}}$ & & $1.111^{\mathrm{v}}$ \\
\hline & & & $0.94360^{t}$ & & & & \\
\hline & & & $0.95177^{1}$ & & & & \\
\hline & & & $0.94355^{\mathrm{n}}$ & & & & \\
\hline & 313.15 & 0.9394 & $0.93980^{\mathrm{e}}$ & 1422.9 & $1431.27^{\mathrm{t}}$ & 1.036 & $1.020^{\mathrm{e}}$ \\
\hline & & & $0.93930^{t}$ & & $1431.00^{9}$ & & $0.982^{u}$ \\
\hline & & & $0.93940^{\mathrm{n}}$ & & & & $1.01^{\mathrm{n}}$ \\
\hline & & & $0.93850^{\mathrm{n}}$ & & & & \\
\hline & & & $0.93970^{q}$ & & & & \\
\hline & 318.15 & 0.9353 & $0.93570^{\mathrm{e}}$ & 1402.1 & $1412.74^{t}$ & 0.966 & $0.968^{\mathrm{e}}$ \\
\hline & & & $0.93510^{t}$ & & & & \\
\hline
\end{tabular}

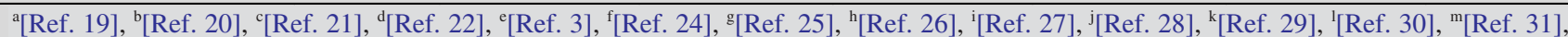

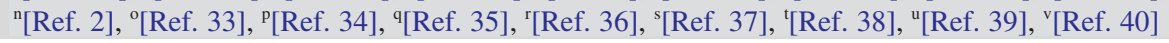

\section{RESULTS AND DISCUSSION}

Fourier transform Infrared spectroscopy used to determine the position and absorption of peaks in the infrared region from 4000 to $400 \mathrm{~cm}^{-1}$. Excess parameters in terms of thermodynamic studies are necessary to estimate the nature, extent and type of interactions/intermolecular forces between the liquid components in binary mixtures. Deviation from ideal behaviour arises due to the interactions of one component with the other component in the liquid mixtures.
$p$-Chloroacetophenone is a protic solvent with dipole moment $1.5549 \mu$ and amines are strongly polar as they have large dipole moments. Primary and secondary amines form hydrogen bonding but tertiary amines do not, because they have no N-H bonds. Experimental values of density, viscosity and speed of sound at all mole fraction range and at temperatures of 303.15, $308.15,313.15,318.15 \mathrm{~K}$ of binary mixtures (PCAP+AN), (PCAP+NMA), (PCAP+NNDMA) is presented in Table-3.

Experimental values of excess molar volumes $\left(\mathrm{V}^{\mathrm{E}}\right)$, deviation in viscosity $(\Delta \eta)$, deviation in isentropic compressibility

TABLE-3

DENSITIES $\left(\rho, \mathrm{g} \mathrm{cm}^{-3}\right)$, ULTRASONIC VELOCITIES $\left(\mathrm{u}, \mathrm{m} \mathrm{s}^{-1}\right)$ AND VISCOSITIES $(\eta, \mathrm{mPa} \mathrm{s})$ OF BINARY MIXTURES OF $p$-CHLOROACETOPHENONE WITH ANILINES AND $N$-ALKYL ANILINES AT DIFFERENT TEMPERATURES (303.15, 308.15, 313.15 AND 318.15 K)

\begin{tabular}{|c|c|c|c|c|c|c|c|c|c|c|c|c|}
\hline \multirow{2}{*}{$\mathrm{x}_{1}$} & \multicolumn{3}{|c|}{$303.15 \mathrm{~K}$} & \multicolumn{3}{|c|}{$308.15 \mathrm{~K}$} & \multicolumn{3}{|c|}{$313.15 \mathrm{~K}$} & \multicolumn{3}{|c|}{$318.15 \mathrm{~K}$} \\
\hline & $\rho$ & $\mathrm{u}$ & $\eta$ & $\rho$ & $\mathrm{u}$ & $\eta$ & $\rho$ & $\mathrm{u}$ & $\eta$ & $\rho$ & $\mathrm{u}$ & $\eta$ \\
\hline \multicolumn{13}{|c|}{ PCAP + Aniline } \\
\hline 0.0000 & 1.0150 & 1625.40 & 2.9890 & 1.0106 & 1605.00 & 2.6430 & 1.0062 & 1585.30 & 2.2970 & 1.0018 & 1565.40 & 1.9510 \\
\hline 0.0705 & 1.0349 & 1612.83 & 2.8989 & 1.0308 & 1595.97 & 2.5624 & 1.0271 & 1575.26 & 2.2258 & 1.0226 & 1602.84 & 1.8869 \\
\hline 0.1462 & 1.0538 & 1596.69 & 2.8224 & 1.0497 & 1580.41 & 2.5012 & 1.0460 & 1561.40 & 2.1798 & 1.0413 & 1599.37 & 1.8565 \\
\hline 0.2271 & 1.0723 & 1579.35 & 2.7494 & 1.0680 & 1563.16 & 2.4486 & 1.0643 & 1544.41 & 2.1486 & 1.0595 & 1580.44 & 1.8442 \\
\hline 0.3880 & 1.1042 & 1544.47 & 2.6273 & 1.0995 & 1527.16 & 2.3738 & 1.0957 & 1507.93 & 2.1168 & 1.0906 & 1529.58 & 1.8577 \\
\hline 0.4875 & 1.1215 & 1523.20 & 2.5606 & 1.1166 & 1505.40 & 2.3367 & 1.1127 & 1485.92 & 2.1069 & 1.1075 & 1496.59 & 1.8783 \\
\hline 0.5879 & 1.1374 & 1502.28 & 2.4982 & 1.1324 & 1484.37 & 2.3019 & 1.1285 & 1464.13 & 2.1021 & 1.1232 & 1464.24 & 1.9020 \\
\hline 0.6894 & 1.1521 & 1481.94 & 2.4384 & 1.1471 & 1463.40 & 2.2716 & 1.1429 & 1442.99 & 2.1026 & 1.1376 & 1432.82 & 1.9319 \\
\hline 0.7919 & 1.1653 & 1461.49 & 2.3877 & 1.1603 & 1442.08 & 2.2523 & 1.1561 & 1421.30 & 2.1149 & 1.1507 & 1402.40 & 1.9746 \\
\hline 0.8954 & 1.1770 & 1439.77 & 2.3520 & 1.1719 & 1418.35 & 2.2518 & 1.1672 & 1397.14 & 2.1483 & 1.1621 & 1372.75 & 2.0415 \\
\hline 1.0000 & 1.1859 & 1412.80 & 2.3538 & 1.1806 & 1389.64 & 2.2924 & 1.1755 & 1366.12 & 2.2314 & 1.1704 & 1343.04 & 2.1704 \\
\hline \multicolumn{13}{|c|}{ PCAP $+N$-Methylaniline } \\
\hline 0.0000 & 0.9806 & 1538.90 & 1.7220 & 0.9765 & 1518.00 & 1.5670 & 0.9724 & 1498.50 & 1.4120 & 0.9683 & 1478.02 & 1.2570 \\
\hline 0.0827 & 1.0030 & 1530.42 & 1.7316 & 0.9991 & 1512.33 & 1.5756 & 0.9952 & 1493.06 & 1.4178 & 0.9910 & 1511.49 & 1.2597 \\
\hline 0.1690 & 1.0250 & 1521.05 & 1.7602 & 1.0210 & 1502.87 & 1.6064 & 1.0171 & 1483.67 & 1.4555 & 1.0130 & 1511.82 & 1.2993 \\
\hline 0.2645 & 1.0480 & 1509.63 & 1.7987 & 1.0438 & 1491.03 & 1.6530 & 1.0399 & 1471.47 & 1.5070 & 1.0355 & 1498.95 & 1.3591 \\
\hline 0.4296 & 1.0847 & 1489.18 & 1.8874 & 1.0801 & 1470.00 & 1.7587 & 1.0761 & 1449.63 & 1.6247 & 1.0715 & 1466.01 & 1.4930 \\
\hline 0.5305 & 1.1054 & 1476.49 & 1.9486 & 1.1006 & 1457.08 & 1.8304 & 1.0966 & 1436.46 & 1.7056 & 1.0919 & 1444.02 & 1.5837 \\
\hline 0.6289 & 1.1246 & 1464.38 & 2.0126 & 1.1198 & 1444.99 & 1.9040 & 1.1156 & 1423.82 & 1.7906 & 1.1107 & 1422.44 & 1.6782 \\
\hline 0.7250 & 1.1424 & 1452.59 & 2.0808 & 1.1375 & 1432.83 & 1.9824 & 1.1331 & 1411.52 & 1.8791 & 1.1282 & 1401.69 & 1.7742 \\
\hline 0.8188 & 1.1587 & 1500.66 & 2.1567 & 1.1538 & 1420.43 & 2.0664 & 1.0490 & 1462.32 & 1.9746 & 1.1443 & 1489.52 & 1.4169 \\
\hline 0.9104 & 1.1734 & 1427.89 & 2.2432 & 1.1684 & 1406.84 & 2.1660 & 1.1636 & 1384.35 & 2.0862 & 1.1587 & 1362.32 & 2.0049 \\
\hline 1.0000 & 1.1859 & 1412.80 & 2.3538 & 1.1806 & 1389.64 & 2.2924 & 1.1755 & 1366.12 & 2.2314 & 1.1704 & 1343.04 & 2.1704 \\
\hline
\end{tabular}




\begin{tabular}{llll|lll|llllll}
\hline \multicolumn{10}{c}{ PCAP $+N, N$-Dimethylaniline } \\
\hline 0.0000 & 0.9481 & 1462.80 & 1.1760 & 0.9435 & 1442.30 & 1.1060 & 0.9394 & 1422.90 & 1.0360 & 0.9353 & 1402.10 & 0.9660 \\
0.0955 & 0.9730 & 1459.59 & 1.2493 & 0.9686 & 1440.62 & 1.1715 & 0.9647 & 1422.00 & 1.0937 & 0.9607 & 1434.58 & 1.0156 \\
0.1924 & 0.9980 & 1456.13 & 1.3379 & 0.9935 & 1437.13 & 1.2566 & 0.9895 & 1418.41 & 1.1794 & 0.9855 & 1439.93 & 1.0962 \\
0.2901 & 1.0228 & 1452.44 & 1.4362 & 1.0181 & 1432.70 & 1.3549 & 1.0141 & 1413.81 & 1.2768 & 1.0099 & 1434.78 & 1.1937 \\
0.4685 & 1.0666 & 1444.27 & 1.6313 & 1.0617 & 1423.79 & 1.5537 & 1.0574 & 1404.25 & 1.4787 & 1.0529 & 1415.88 & 1.3957 \\
0.5695 & 1.0906 & 1439.35 & 1.7481 & 1.0857 & 1418.55 & 1.6729 & 1.0812 & 1398.77 & 1.5984 & 1.0767 & 1402.93 & 1.5174 \\
0.6650 & 1.1128 & 1434.51 & 1.8648 & 1.1079 & 1413.66 & 1.7903 & 1.1034 & 1393.69 & 1.7181 & 1.0988 & 1390.21 & 1.6364 \\
0.7552 & 1.1333 & 1429.80 & 1.9817 & 1.1284 & 1409.19 & 1.9074 & 1.1238 & 1388.90 & 1.8346 & 1.1193 & 1377.95 & 1.7553 \\
0.8411 & 1.1525 & 1424.64 & 2.1006 & 1.1475 & 1403.85 & 2.0272 & 1.1429 & 1382.96 & 1.9565 & 1.1383 & 1365.99 & 1.8785 \\
0.9226 & 1.1700 & 1419.36 & 2.2232 & 1.1650 & 1398.17 & 2.1534 & 1.1603 & 1376.69 & 2.0825 & 1.1556 & 1354.49 & 2.0128 \\
1.0000 & 1.1859 & 1412.80 & 2.3538 & 1.1806 & 1389.64 & 2.2924 & 1.1755 & 1366.12 & 2.2314 & 1.1704 & 1343.04 & 2.1704 \\
\hline
\end{tabular}

$\left(\Delta \mathrm{k}_{\mathrm{s}}\right)$, Grunberg-Nissan interaction parameters (d), Katti-Chaudari interaction parameters (Wvis) and hind interaction parameters $\left(\mathrm{H}_{12}\right)$ at temperatures $(303.15,308.15,313.15,318.15) \mathrm{K}$ over the entire composition range of (PCAP + AN, PCAP + NMA, PCAP + NNDMA) are represented in Table- 4 . Further, the values obtained from excess properties with Redlich-Kister polynomial equation were also correlated.

Excess molar volume $\left(\mathbf{V}^{\mathrm{E}}\right)$ : The excess molar volume $\left(\mathrm{V}^{\mathrm{E}}\right)$ of liquid mixtures depends on the size and shape of the molecules and the intermolecular forces in a given system [42]. Fig. 1 shows that $\mathrm{V}^{\mathrm{E}}$ for $p$-chloroacetophenone with aniline, $\mathrm{N}$-methylaniline and $\mathrm{N}, \mathrm{N}$-dimethylaniline are negative over the entire composition range. The excess molar volume negative data may be due to three factors [43]:

(a) Chemical association between unlike molecules through the formation of hydrogen bond, which is a strong specific interaction;

TABLE-4

EXPERIMENTAL VALUES OF EXCESS MOLAR VOLUMES $\left(\mathrm{V}^{\mathrm{E}}, \mathrm{cm}^{3} \mathrm{~mol}^{-1}\right)$, DEVIATION IN VISCOSITY $(\Delta \eta, \mathrm{mPa} s)$, DEVIATION IN ISENTROPIC COMPRESSIBILITY $\left(\Delta \mathrm{k}_{\mathrm{s}},\left(\mathrm{p} \mathrm{a}^{-1}\right)\right.$, GRUNBERG-NISSAN INTERACTION PARAMETERS (d), KATTI-CHAUDARI INTERACTION PARAMETERS (Wvis) AND HIND INTERACTION PARAMETERS $\left(\mathrm{H}_{12}\right)$ AT DIFFERENT TEMPERATURE

\begin{tabular}{|c|c|c|c|c|c|c|c|c|c|c|c|c|}
\hline \multirow{2}{*}{$\mathrm{x}_{1}$} & $V^{E}$ & $\Delta \mathrm{k}_{\mathrm{s}}$ & $\Delta \eta$ & $\mathrm{d}$ & $\mathrm{W}_{\text {vis }}$ & $\mathrm{H}_{12}$ & $\mathrm{~V}^{\mathrm{E}}$ & $\Delta \mathrm{k}_{\mathrm{s}}$ & $\Delta \eta$ & $\mathrm{d}$ & $\mathrm{W}_{\text {vis }}$ & $\mathrm{H}_{12}$ \\
\hline & \multicolumn{6}{|c|}{ PCAP + Aniline $(303.15 \mathrm{~K})$} & \multicolumn{6}{|c|}{ PCAP + Aniline $(308.15 \mathrm{~K})$} \\
\hline 0.0000 & 0.0000 & 0.0000 & 0.0000 & 0.0000 & 0.0000 & 0.0000 & 0.0000 & 0.0000 & 0.0000 & 0.0000 & 0.0000 & 0.0000 \\
\hline 0.0705 & -0.3020 & -0.4954 & -0.0454 & -0.0913 & -0.0829 & 2.3253 & -0.3378 & -0.7101 & -0.0559 & -0.1388 & -0.1327 & 2.0413 \\
\hline 0.1462 & -0.4991 & -0.7953 & -0.0737 & -0.0780 & -0.0668 & 2.3762 & -0.5442 & -1.0680 & -0.0905 & -0.1195 & -0.1098 & 2.1050 \\
\hline 0.2271 & -0.6560 & -1.0303 & -0.0954 & -0.0725 & -0.0603 & 2.3997 & -0.6900 & -1.3292 & -0.1148 & -0.1091 & -0.0976 & 2.1406 \\
\hline 0.3880 & -0.7984 & -1.2500 & -0.1152 & -0.0663 & -0.0527 & 2.4288 & -0.8142 & -1.5301 & -0.1332 & -0.0955 & -0.0820 & 2.1873 \\
\hline 0.4875 & -0.8173 & -1.2762 & -0.1188 & -0.0665 & -0.0526 & 2.4337 & -0.8267 & -1.5515 & -0.1354 & -0.0935 & -0.0798 & 2.1968 \\
\hline 0.5879 & -0.8059 & -1.2492 & -0.1174 & -0.0698 & -0.0563 & 2.4291 & -0.8116 & -1.5388 & -0.1350 & -0.0977 & -0.0843 & 2.1891 \\
\hline 0.6894 & -0.7520 & -1.1836 & -0.1127 & -0.0789 & -0.0663 & 2.4083 & -0.7654 & -1.4607 & -0.1297 & -0.1081 & -0.0957 & 2.1649 \\
\hline 0.7919 & -0.6430 & -1.0406 & -0.0983 & -0.0934 & -0.0823 & 2.3731 & -0.6599 & -1.2855 & -0.1130 & -0.1245 & -0.1137 & 2.1248 \\
\hline 0.8954 & -0.4384 & -0.7421 & -0.0683 & -0.1195 & -0.1112 & 2.3070 & -0.4542 & -0.8743 & -0.0773 & -0.1519 & -0.1441 & 2.0551 \\
\hline \multirow[t]{2}{*}{1.0000} & 0.0000 & 0.0000 & 0.0000 & 0.0000 & 0.0000 & 0.0000 & 0.0000 & 0.0000 & 0.0000 & 0.0000 & 0.0000 & 0.0000 \\
\hline & \multicolumn{6}{|c|}{ PCAP $+N$-Methylaniline $(303.15 \mathrm{~K})$} & \multicolumn{6}{|c|}{ PCAP $+N$-Methylaniline $(308.15 \mathrm{~K})$} \\
\hline 0.0000 & 0.0000 & 0.0000 & 0.0000 & 0.0000 & 0.0000 & 0.0000 & 0.0000 & 0.0000 & 0.0000 & 0.0000 & 0.0000 & 0.0000 \\
\hline 0.0827 & -0.2717 & -0.4221 & -0.0426 & -0.1160 & -0.1229 & 1.7571 & -0.3074 & -0.6302 & -0.0514 & -0.1489 & -0.1576 & 1.5907 \\
\hline 0.1690 & -0.4721 & -0.7467 & -0.0686 & -0.0956 & -0.1015 & 1.7936 & -0.5105 & -0.9775 & -0.0832 & -0.1220 & -0.1289 & 1.6336 \\
\hline 0.2645 & -0.6290 & -0.9657 & -0.0904 & -0.0872 & -0.0925 & 1.8056 & -0.6629 & -1.1941 & -0.1058 & -0.1053 & -0.1112 & 1.6577 \\
\hline 0.4296 & -0.7546 & -1.1285 & -0.1060 & -0.0754 & -0.0799 & 1.8216 & -0.7671 & -1.3464 & -0.1199 & -0.0851 & -0.0897 & 1.6850 \\
\hline 0.5305 & -0.7600 & -1.1230 & -0.1085 & -0.0735 & -0.0778 & 1.8200 & -0.7661 & -1.3403 & -0.1214 & -0.0810 & -0.0853 & 1.6859 \\
\hline 0.6289 & -0.7250 & -1.0739 & -0.1068 & -0.0756 & -0.0800 & 1.8092 & -0.7374 & -1.3069 & -0.1192 & -0.0827 & -0.0872 & 1.6744 \\
\hline 0.7250 & -0.6576 & -0.9776 & -0.0993 & -0.0813 & -0.0863 & 1.7889 & -0.6745 & -1.2006 & -0.1105 & -0.0885 & -0.0937 & 1.6527 \\
\hline 0.8188 & -0.5419 & -0.8091 & -0.0827 & -0.0903 & -0.0963 & 1.7594 & -0.5622 & -1.0098 & -0.0946 & -0.1020 & -0.1085 & 1.6110 \\
\hline 0.9104 & -0.3440 & -0.5178 & -0.0539 & -0.1070 & -0.1149 & 1.7072 & -0.3732 & -0.6720 & -0.0614 & -0.1205 & -0.1295 & 1.5533 \\
\hline \multirow[t]{2}{*}{1.0000} & & 0.0000 & 0.0000 & 0.0000 & 0.0000 & 0.0000 & 0.0000 & 0.0000 & 0.0000 & 0.0000 & 0.0000 & 0.0000 \\
\hline & \multicolumn{6}{|c|}{ PCAP $+N, N$-Dimethylaniline $(303.15 \mathrm{~K})$} & \multicolumn{6}{|c|}{ PCAP $+N, N$-Dimethylaniline $(308.15 \mathrm{~K})$} \\
\hline 0.0000 & 0.0000 & 0.0000 & 0.0000 & 0.0000 & 0.0000 & 0.0000 & 0.0000 & 0.0000 & 0.0000 & 0.0000 & 0.0000 & 0.0000 \\
\hline 0.0955 & -0.2401 & -0.3794 & -0.0392 & -0.0293 & -0.0387 & 1.5379 & -0.2753 & -0.5284 & -0.0478 & -0.0605 & -0.0712 & 1.4227 \\
\hline 0.1924 & -0.4435 & -0.6814 & -0.0647 & -0.0127 & -0.0222 & 1.5567 & -0.4755 & -0.8518 & -0.0776 & -0.0350 & -0.0453 & 1.4494 \\
\hline 0.2901 & -0.5960 & -0.9025 & -0.0815 & -0.0030 & -0.0127 & 1.5671 & -0.6248 & -1.0448 & -0.0953 & -0.0178 & -0.0280 & 1.4679 \\
\hline 0.4685 & -0.7161 & -1.0453 & -0.0965 & 0.0038 & -0.0057 & 1.5712 & -0.7353 & -1.1683 & -0.1081 & -0.0027 & -0.0126 & 1.4821 \\
\hline 0.5695 & -0.7057 & -1.0225 & -0.0987 & 0.0022 & -0.0074 & 1.5637 & -0.7248 & -1.1417 & -0.1087 & -0.0022 & -0.0121 & 1.4775 \\
\hline 0.6650 & -0.6417 & -0.9382 & -0.0944 & -0.0008 & -0.0103 & 1.5530 & -0.6737 & -1.0721 & -0.1046 & -0.0059 & -0.0159 & 1.4644 \\
\hline 0.7552 & -0.5467 & -0.8104 & -0.0838 & -0.0052 & -0.0149 & 1.5383 & -0.5787 & -0.9709 & -0.0946 & -0.0128 & -0.0231 & 1.4434 \\
\hline 0.8411 & -0.4216 & -0.6143 & -0.0660 & -0.0115 & -0.0218 & 1.5179 & -0.4537 & -0.7705 & -0.0766 & -0.0231 & -0.0343 & 1.4124 \\
\hline 0.9226 & -0.2454 & -0.3673 & -0.0395 & -0.0206 & -0.0319 & 1.4885 & -0.2774 & -0.5036 & -0.0471 & -0.0372 & -0.0500 & 1.3692 \\
\hline 1.0000 & 0.0000 & 0.0000 & 0.0000 & 0.0000 & 0.0000 & 0.0000 & 0.0000 & 0.0000 & 0.0000 & 0.0000 & 0.0000 & 0.0000 \\
\hline
\end{tabular}




\begin{tabular}{|c|c|c|c|c|c|c|c|c|c|c|c|c|}
\hline & \multicolumn{6}{|c|}{ PCAP + Aniline (313.15 K) } & \multicolumn{6}{|c|}{ PCAP + Aniline (318.15 K) } \\
\hline 0.0000 & 0.0000 & 0.0000 & 0.0000 & 0.0000 & 0.0000 & 0.0000 & 0.0000 & 0.0000 & 0.0000 & 0.0000 & 0.0000 & 0.0000 \\
\hline 0.0705 & -0.4096 & -0.7317 & -0.0665 & -0.1950 & -0.1933 & 1.7566 & -0.4146 & -0.9123 & -0.0795 & -0.3014 & -0.3221 & 1.1898 \\
\hline 0.1462 & -0.6284 & -1.2106 & -0.1076 & -0.1675 & -0.1602 & 1.8331 & -0.6177 & -1.4180 & -0.1265 & -0.2207 & -0.2362 & 1.2898 \\
\hline 0.2271 & -0.7856 & -1.5191 & -0.1335 & -0.1490 & -0.1392 & 1.8839 & -0.7745 & -1.7730 & -0.1566 & -0.1693 & -0.1828 & 1.3504 \\
\hline 0.3880 & -0.9126 & -1.7450 & -0.1548 & -0.1289 & -0.1165 & 1.9384 & -0.8808 & -2.0823 & -0.1784 & -0.1079 & -0.1188 & 1.4209 \\
\hline 0.4875 & -0.9249 & -1.7801 & -0.1582 & -0.1257 & -0.1129 & 1.9477 & -0.8913 & -2.1111 & -0.1797 & -0.0900 & -0.1002 & 1.4370 \\
\hline 0.5879 & -0.9111 & -1.7512 & -0.1564 & -0.1284 & -0.1160 & 1.9415 & -0.8751 & -2.1039 & -0.1780 & -0.0857 & -0.0960 & 1.4293 \\
\hline 0.6894 & -0.8516 & -1.6821 & -0.1492 & -0.1388 & -0.1273 & 1.9159 & -0.8246 & -2.0348 & -0.1703 & -0.0920 & -0.1030 & 1.3989 \\
\hline 0.7919 & -0.7498 & -1.5046 & -0.1302 & -0.1572 & -0.1478 & 1.8693 & -0.7127 & -1.8306 & -0.1501 & -0.1085 & -0.1210 & 1.3412 \\
\hline 0.8954 & -0.5002 & -1.0590 & -0.0899 & -0.1900 & -0.1833 & 1.7841 & -0.4 & -1.3327 & -0.1059 & -0.1427 & -0.1584 & 1.2311 \\
\hline \multirow[t]{2}{*}{1.0000} & 000 & 0.0000 & 0.0000 & 0.0000 & 0.0000 & 0.0000 & 0.0000 & 0.0000 & 0.0000 & 0.0000 & 0.0000 & 0.0000 \\
\hline & \multicolumn{6}{|c|}{ PCAP $+N$-Methylaniline $(313.15 \mathrm{~K})$} & \multicolumn{6}{|c|}{ PCAP + N-Methylaniline (318.15 K) } \\
\hline 0.0000 & & 0.0000 & 0.0000 & 0.0000 & 0.0000 & & & 0.0000 & 0.0000 & 0.0000 & 0.0000 & 0.0000 \\
\hline 0.0827 & -0.3406 & -0.7028 & -0.0620 & -0.1932 & -0.2018 & 1.4133 & -0.3507 & -0.8432 & -0.0728 & -0.2165 & -0.2273 & 1.5492 \\
\hline 0.1690 & -0.5631 & -1.0964 & -0.0950 & -0.1453 & -0.1518 & 1.4836 & -0.5893 & -1.3139 & -0.1121 & -0.1713 & -0.1803 & 1.6301 \\
\hline 0.2645 & -0.7231 & -1.3270 & -0.1218 & -0.1249 & -0.1301 & 1.5088 & -0.7351 & -1.6162 & -0.1395 & -0.1467 & -0.1539 & 1.6706 \\
\hline 0.4296 & -0.8396 & -1.4840 & -0.1393 & -0.0998 & -0.1037 & 1.5374 & -0.8387 & -1.8204 & -0.1564 & -0.1201 & -0.1258 & 1.7100 \\
\hline 0.5305 & -0.8423 & -1.4900 & -0.1411 & -0.0939 & -0.0975 & 1.5384 & -0.8414 & -1.8357 & -0.1578 & -0.1146 & -0.1200 & 1.7123 \\
\hline 0.6289 & -0.8114 & -1.4474 & -0.1368 & -0.0935 & 0974 & 5287 & -0.8072 & -1.7843 & -0.1533 & -0.1152 & -0.1208 & 1.7008 \\
\hline 0.7250 & -0.7336 & -1.3475 & -0.1270 & -0.1002 & -0.1046 & 1.5032 & -0.7393 & -1.6937 & -0.1450 & -0.1256 & -0.1319 & 1.6656 \\
\hline 0.8188 & -0.6072 & -1.1505 & -0.1083 & -0.1152 & -0.1208 & 1.4566 & -0.6240 & -1.4884 & -0.1243 & -0.1429 & -0.1508 & 1.6102 \\
\hline 0.9104 & -0.4037 & -0.7600 & -0.0718 & -0.1399 & 1483 & 3817 & -0.4171 & -1.0182 & -0.0837 & -0.1732 & -0.1840 & 1.5161 \\
\hline \multirow[t]{2}{*}{1.0000} & 0.0000 & 0.0000 & 0.0000 & 0.0000 & 0.0000 & 0.0000 & 0.0000 & 0.0000 & 0.0000 & 0.0000 & 0.0000 & 0.0000 \\
\hline & \multicolumn{6}{|c|}{ PCAP $+N, N$-Dimethylaniline $(313.15 \mathrm{~K})$} & \multicolumn{6}{|c|}{ PCAP $+N, N$-Dimethylaniline $(318.15 \mathrm{~K})$} \\
\hline 0.0000 & 0.0000 & 0.0000 & 0.0000 & 0.0000 & 0.0000 & 0.0000 & 0.0000 & 0.0000 & 0.0000 & 0.0000 & 0.0000 & 0.0000 \\
\hline 0.0955 & -0.3105 & -0.6438 & -0.0565 & -0.0961 & -0.1081 & 1.3066 & -0.3508 & -0.7662 & -0.0655 & -0.1371 & -0.1506 & 1.1893 \\
\hline 0.1924 & -0.5172 & -0.9994 & -0.0866 & -0.0503 & -0.0614 & 1.3550 & -0.5576 & -1.2113 & -0.1015 & -0.0818 & -0.0938 & 1.2416 \\
\hline 0.2901 & -0.6701 & -1.2136 & -0.1060 & -0.0286 & -0.0395 & 1.3764 & -0.7104 & -1.4279 & -0.1217 & -0.0490 & -0.0604 & 1.2726 \\
\hline 0.4685 & -0.7679 & -1.3403 & -0.1173 & -0.0064 & -0.0166 & 1.3981 & -0.7913 & -1.5733 & -0.1345 & -0.0196 & -0.0301 & 1.2981 \\
\hline 0.5695 & -0.7570 & -1.3243 & -0.1184 & -0.0059 & -0.0162 & 1.3922 & -0.7838 & -1.5480 & -0.1345 & -0.0166 & -0.0272 & 1.2940 \\
\hline 0.6650 & -0.7059 & -1.2662 & -0.1128 & -0.0085 & -0.0190 & 1.3805 & -0.7428 & -1.4990 & -0.1306 & -0.0219 & -0.0329 & 1.2751 \\
\hline 0.7552 & -0.6172 & -1.1679 & -0.1041 & -0.0187 & -0.0297 & 1.3520 & -0.6642 & -1.3974 & -0.1202 & -0.0331 & -0.0449 & 1.2430 \\
\hline 0.8411 & -0.4985 & -0.9472 & -0.0850 & -0.0311 & -0.0434 & 1.3158 & -0.5389 & -1.1538 & -0.1005 & -0.0513 & -0.0646 & 1.1922 \\
\hline 0.9226 & -0.3094 & -0.6498 & -0.0564 & -0.0588 & -0.0731 & 1.2390 & -0.3498 & -0.7723 & -0.0644 & -0.0774 & -0.0935 & 1.1173 \\
\hline 1.0000 & 0.0000 & 0.0000 & 0.0000 & 0.0000 & 0.0000 & 0.0000 & 0.0000 & 0.0000 & 0.0000 & 0.0000 & 0.0000 & 0.0000 \\
\hline
\end{tabular}

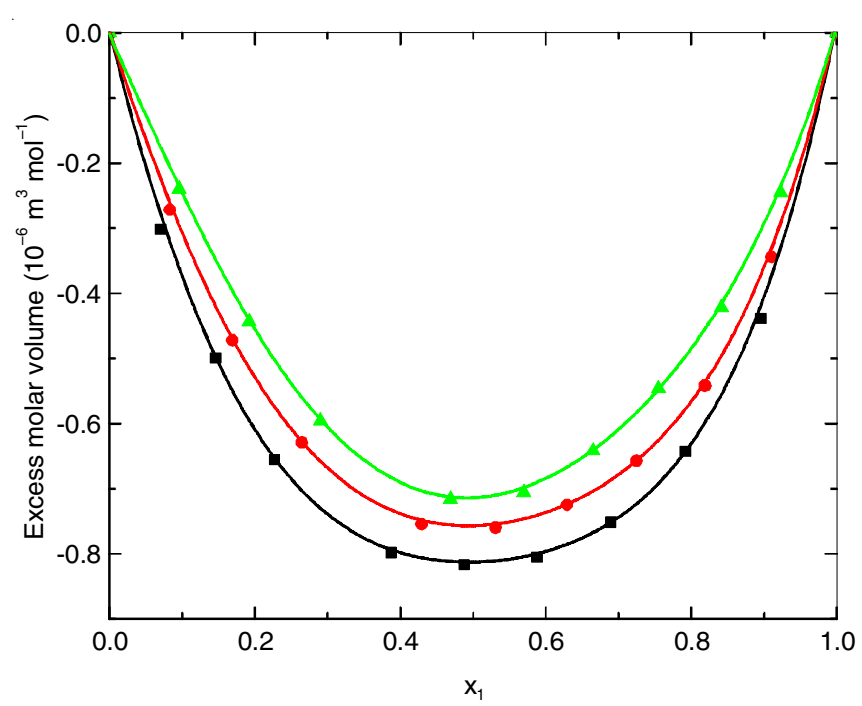

Fig. 1. Variation of excess molar volume $\left(\mathrm{V}^{\mathrm{E}}\right)$ of the binary liquid mixtures of $p$-chloro-acetophenone with aniline ( $), N$-methylaniline ( $)$, $N, N$-dimethylaniline $(\Delta)$ at $303.15 \mathrm{~K}$

(b) Association through weaker physical forces like dipoledipole interaction and,

(c) Accommodation of molecular of one component into the interstitial positions of the structural network of molecules of another component.
Similar kind of trend is also noticed for $\mathrm{N}, \mathrm{N}$-dimethylformamide with ketones [44]. Nitrogen atoms in anilines are good donors due to the lone pair of electrons on it. The strength of interaction order as follows:

Aniline $>N$-Methylaniline $>N, N$-dimethylaniline

$$
\mathrm{V}^{\mathrm{E}}=\frac{\left[\mathrm{x}_{\mathrm{i}} \mathrm{M}_{1}+\mathrm{x}_{2} \mathrm{M}_{2}\right]}{\rho_{\mathrm{m}}-\left(\frac{\mathrm{x}_{1} \mathrm{M}_{1}}{\rho_{1}}+\frac{\mathrm{x}_{2} \mathrm{M}_{2}}{\rho_{2}}\right)}
$$

where, $x_{1}, x_{2}, M_{1}, M_{2}, \rho_{1}, \rho_{2}$ and $\rho_{m}$ represent mole fraction, molecular mass, density of component 1 and 2 and the mixture, respectively.

Excess partial molar volumes: The partial molar volumes, $\mathrm{V}_{\mathrm{m}, 1}^{\circ}$ of component 1 ( $p$-chloroacetophenone $\left(\mathrm{X}_{1}\right)$ ) and $\mathrm{V}_{\mathrm{m}, 2}^{\circ}$ of component 2 (aniline, $N$-methylaniline and N,N-dimethylaniline $\left.\left(\mathrm{X}_{2}\right)\right)$ in the mixture over the entire composition range are calculated using the following relations:

$$
\begin{aligned}
& \mathrm{V}_{\mathrm{m}, 1}^{\mathrm{oE}}=\mathrm{V}_{\mathrm{m}}^{\mathrm{E}}+\mathrm{V}_{\mathrm{m}, \mathrm{1}}^{*}+\mathrm{x}_{2}\left(\frac{\partial \mathrm{V}_{\mathrm{m}}^{\mathrm{E}}}{\partial \mathrm{x}_{1}}\right)_{\mathrm{T}, \mathrm{P}} \\
& \mathrm{V}_{\mathrm{m}, 2}^{\mathrm{oE}}=\mathrm{V}_{\mathrm{m}}^{\mathrm{E}}+\mathrm{V}_{\mathrm{m}, 2}^{*}+\mathrm{x}_{1}\left(\frac{\partial \mathrm{V}_{\mathrm{m}}^{\mathrm{E}}}{\partial \mathrm{x}_{1}}\right)_{\mathrm{T}, \mathrm{P}}
\end{aligned}
$$


where $\mathrm{V}_{\mathrm{m}, 1}^{\circ}$ and $\mathrm{V}_{\mathrm{m}, 2}^{\circ}$ are the molar volumes of pure components, $p$-chloroacetophenone + aniline and substituted anilines. The $\left(\partial \mathrm{V}_{\mathrm{m}}^{\mathrm{E}} / \partial \mathrm{x}_{1}\right)_{\mathrm{T}, \mathrm{P}}$ value in eqns. 1 and 2 was calculated differentiating Redlich-Kister polynomial fitting. The eqns. 1 and 2 partial molar volumes used to calculate the excess partial molar volumes using the following equations:

$$
\begin{aligned}
& \mathrm{V}_{\mathrm{m}, 1}^{\circ, \mathrm{E}}=\mathrm{V}_{\mathrm{m}, 1}^{\circ}-\mathrm{V}_{\mathrm{m}, 1}^{*} \\
& \mathrm{~V}_{\mathrm{m}, 2}^{\circ, \mathrm{E}}=\mathrm{V}_{\mathrm{m}, 2}^{\circ}-\mathrm{V}_{\mathrm{m}, 2}^{*}
\end{aligned}
$$

where $\mathrm{V}_{\mathrm{m}, 1}^{\circ} \mathrm{E}$ and $\mathrm{V}_{\mathrm{m}, 2}^{\circ}$ are the excess partial molar volumes listed in Table-5 and Fig. 2. $\mathrm{V}_{\mathrm{m}, 1}^{\circ, \mathrm{E}, \infty}$ and $\mathrm{V}_{\mathrm{m}, 2}^{\circ, \mathrm{E}, \infty}$ are the excess partial molar volumes at infinite dilution were calculated by the following equations:

$$
\begin{aligned}
& \mathrm{V}_{\mathrm{m}, 2}^{\circ, \mathrm{E}, \infty}=\mathrm{V}_{\mathrm{m}, 2}^{*}+\sum_{\mathrm{i}=0}^{\mathrm{n}} \mathrm{A}_{\mathrm{i}} \\
& \mathrm{V}_{\mathrm{m}, 1}^{\mathrm{O}, \mathrm{E}, \infty}=\mathrm{V}_{\mathrm{m}, 1}^{*}+\sum_{\mathrm{i}=0}^{\mathrm{n}} \mathrm{A}_{\mathrm{i}}
\end{aligned}
$$

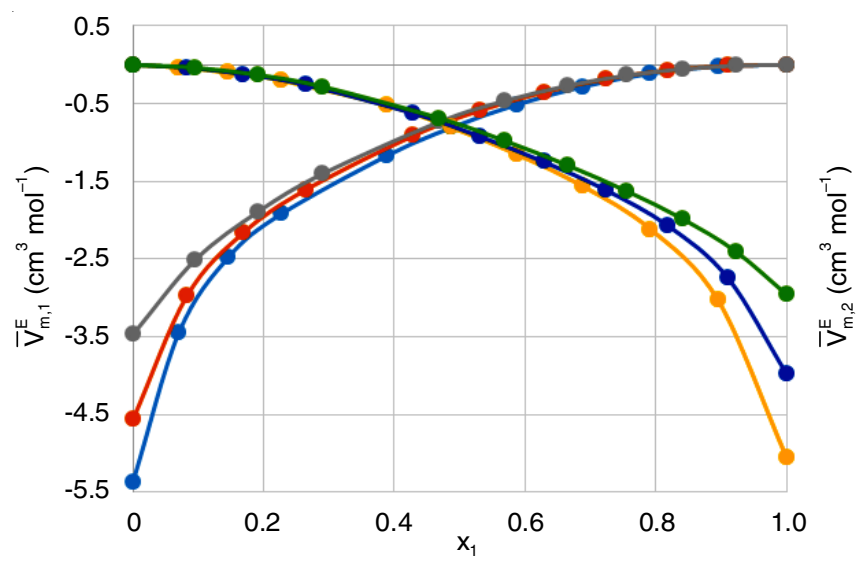

Fig. 2. Excess partial molar volumes of $p$-chloroacetophenone with aniline, $\mathrm{N}$-methylaniline and $\mathrm{N}, \mathrm{N}$-dimethylaniline at $303.15 \mathrm{~K}$

The negative excess partial molar volumes may be indicative of solute-solvent $[45,46]$ interactions between unlike molecules, whereas positive values indicate the presence of strong self-association between like molecules. The observed excess partial molar volume value indicates cross-associations are strong compared to self-associations in $p$-chloroacetophenone
+ aniline and substituted anilines. Most negative values of $\mathrm{V}_{\mathrm{m}, 1}^{\circ, \mathrm{E}, \infty}, \mathrm{V}_{\mathrm{m}, 2}^{\circ, \mathrm{E}, \infty}$ of $p$-chloroacetophenone + aniline and substituted anilines shows stronger solute-solvent interactions at infinite dilution.

Deviation in isentropic compressibility $\left(\Delta \mathbf{k}_{\mathrm{s}}\right)$ : Fig. 3 shows that $\Delta \mathrm{k}_{\mathrm{s}}$ for $p$-chloroacetophenone with aniline, $\mathrm{N}$-methylaniline and $N, N$-dimethylaniline are negative over the entire composition range. The deviation in isentropic compressibility shown negative due to tightly packed molecules, which in turn proves the existence of strong intermolecular interaction between the unlike molecules [47]. $\Delta \mathrm{k}_{\mathrm{s}}$ plays a key role in evaluating the compactness in liquid mixtures through hydrogen bonding, charge-transfer complex formation, dipole-dipole interactions, dipole induced dipole interactions [48].

$$
\begin{gathered}
\mathrm{k}_{\mathrm{s}}=\frac{1}{\rho \mathrm{U}^{2}} \\
\Delta \mathrm{k}_{\mathrm{s}}=\mathrm{k}_{\mathrm{s}}-\left[\mathrm{x}_{1} \mathrm{k}_{\mathrm{s}} 1+\mathrm{x}_{2} \mathrm{k}_{\mathrm{s}}\right]
\end{gathered}
$$

where, $\mathrm{x}_{1}, \mathrm{x}_{2}, \mathrm{k}_{\mathrm{s}} 1, \mathrm{k}_{\mathrm{s}} 2, \mathrm{k}_{\mathrm{s}}$ represent mole fraction, isentropic compressibility of component 1 and 2 and the mixture, respectively.

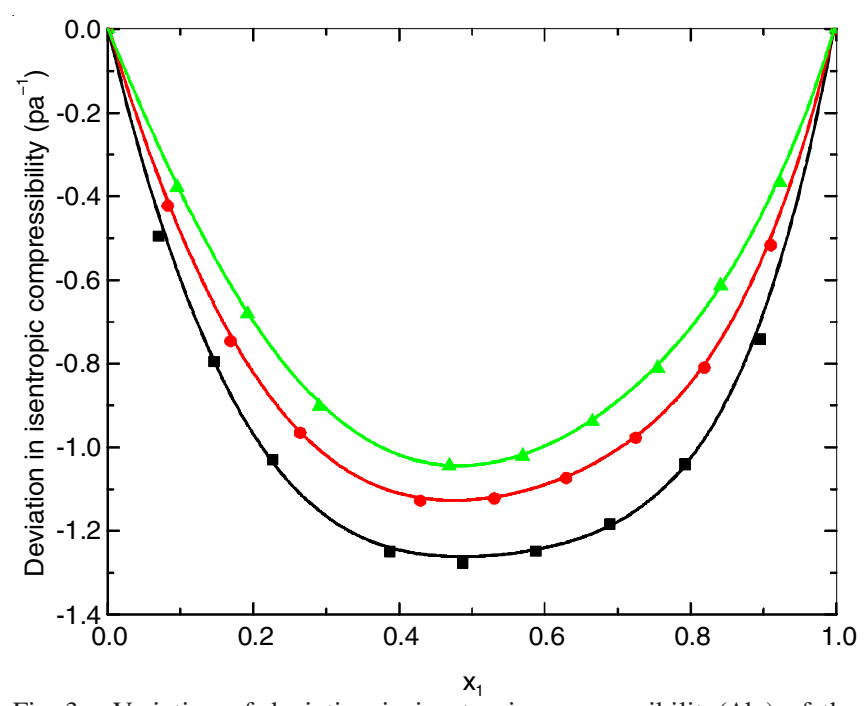

Fig. 3. Variation of deviation in isentropic compressibility $\left(\Delta \mathrm{k}_{\mathrm{s}}\right)$ of the

\begin{tabular}{|c|c|c|c|c|c|c|}
\hline Temp. (K) & $\mathrm{V}_{\mathrm{m}, 1}^{\mathrm{o}, \infty}\left(\mathrm{cm}^{3} \mathrm{~mol}^{-1}\right)$ & $\mathrm{V}_{\mathrm{m}, 1}^{*}\left(\mathrm{~cm}^{3} \mathrm{~mol}^{-1}\right)$ & $\mathrm{V}_{\mathrm{m}, 1}^{\mathrm{o}, \mathrm{E}, \infty}\left(\mathrm{cm}^{3} \mathrm{~mol}^{-1}\right)$ & $\mathrm{V}_{\mathrm{m}, 2}^{\mathrm{o}, \infty}\left(\mathrm{cm}^{3} \mathrm{~mol}^{-1}\right)$ & $\mathrm{V}_{\mathrm{m}, 2}^{*}\left(\mathrm{~cm}^{3} \mathrm{~mol}^{-1}\right)$ & $\mathrm{V}_{\mathrm{m}, 2}^{\mathrm{o}, \mathrm{E}, \infty}\left(\mathrm{cm}^{3} \mathrm{~mol}^{-1}\right)$ \\
\hline \multicolumn{7}{|c|}{ PCAP + Aniline } \\
\hline 303.15 & 124.983 & 130.358 & -5.375 & 86.700 & 91.754 & -5.053 \\
\hline 308.15 & 125.227 & 130.943 & -5.716 & 86.449 & 92.153 & -5.704 \\
\hline 313.15 & 125.035 & 131.512 & -6.477 & 85.729 & 92.556 & -6.827 \\
\hline 318.15 & 125.612 & 132.086 & -6.475 & 86.037 & 92.963 & -6.926 \\
\hline \multicolumn{7}{|c|}{ PCAP $+N$-Methylaniline } \\
\hline 303.15 & 125.789 & 130.358 & -4.569 & 105.298 & 109.276 & -3.978 \\
\hline 308.15 & 125.889 & 130.943 & -5.054 & 105.125 & 109.735 & -4.609 \\
\hline 313.15 & 126.458 & 131.512 & -5.055 & 105.251 & 110.197 & -4.947 \\
\hline 318.15 & 126.384 & 132.086 & -5.703 & 105.314 & 110.664 & -5.350 \\
\hline \multicolumn{7}{|c|}{ PCAP $+N, N$-Dimethylaniline } \\
\hline 303.15 & 126.885 & 130.358 & -3.472 & 124.861 & 127.817 & -2.956 \\
\hline 308.15 & 126.914 & 130.943 & -4.029 & 124.932 & 128.440 & -3.508 \\
\hline 313.15 & 126.918 & 131.512 & -4.595 & 124.927 & 129.000 & -4.073 \\
\hline 318.15 & 130.623 & 132.086 & -1.464 & 128.664 & 129.566 & -0.902 \\
\hline
\end{tabular}
binary liquid mixtures of $p$-chloroacetophenone with aniline ( $\mathbf{\square})$,

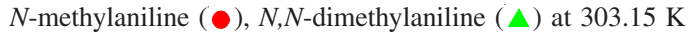

\section{TABLE-5}

\section{VALUES OF $\mathrm{V}_{\mathrm{m}, 1}^{\mathrm{o}, \infty}, \mathrm{V}_{\mathrm{m}, 1}^{*}, \mathrm{~V}_{\mathrm{m}, 1}^{\mathrm{o}, \mathrm{E}, \infty}, \mathrm{V}_{\mathrm{m}, 2}^{\mathrm{o}, \infty}, \mathrm{V}_{\mathrm{m}, 2}^{*}, \mathrm{~V}_{\mathrm{m}, 2}^{\mathrm{o}, \mathrm{E}, \infty}$ OF THE COMPONENTS FOR}

PCAP + ANILINE BINARY SOLUTIONS AT 303.15-318.15 K 
Deviation in viscosity $(\Delta \eta)$ : Fig. 4 shows that $\Delta \eta$ for $p$ chloroacetophenone with aniline, $\mathrm{N}$-methylaniline and $\mathrm{N}, \mathrm{N}$ dimethylaniline are negative over the entire composition range. The negative deviation indicates that there are specific interactions in unlike molecules dominates over the mutual loss of specific interactions between the like molecules [49].

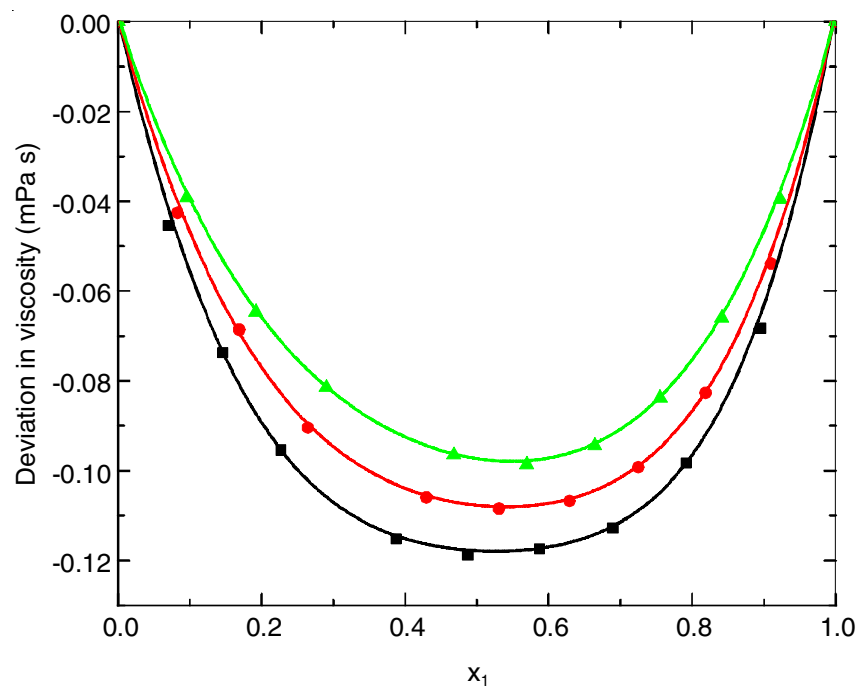

Fig. 4. Variation of deviation in viscosity $(\Delta \eta)$ of the binary liquid mixtures of $p$-chloroacetophenone with aniline $(\boldsymbol{\square}), N$-methylaniline $(\bullet)$, $N, N$-dimethylaniline $(\mathbf{\Delta})$ at $303.15 \mathrm{~K}$

The strength of interaction fall in the order:

Aniline $>N$-Methylaniline $>N, N$-dimethylaniline

Figs. 1 and 4 show that $\mathrm{V}^{\mathrm{E}}$ and $\Delta \eta$ are in the same trend and do not obey the general rule [21].

$$
\Delta \eta=\eta-\left[x_{1} \eta_{1}+x_{2} \eta_{2}\right]
$$

Redlich-Kister equation $\left(\mathbf{Y}^{\mathrm{E}}\right)$ [50]: This equation can be expressed as follows:

$$
\begin{gathered}
Y^{\mathrm{E}}=\mathrm{x}_{1} \mathrm{X}_{2}\left[\mathrm{a}_{0}+\mathrm{a}_{1}\left(\mathrm{x}_{1}-\mathrm{x}_{2}\right)+\mathrm{a}_{2}\left(\mathrm{x}_{1}-\mathrm{x}_{2}\right)^{2}\right] \\
\sigma\left(\mathrm{Y}^{\mathrm{E}}\right)=\left(\frac{\sum\left(\mathrm{Y}_{\mathrm{obs}}^{\mathrm{E}}-\mathrm{Y}_{\mathrm{cal}}^{\mathrm{E}}\right)^{2}}{(\mathrm{n}-\mathrm{m})}\right)^{1 / 2}
\end{gathered}
$$

where $\mathrm{n}$ is the total no. of experimental points and $\mathrm{m}$ is the no. of coefficients. The values of $a_{0}, a_{1}, a_{2}$ are the coefficients are the standard deviation. From Table-6, we can say that the experimental data measured show good results by observing the values of standard deviation.

Grunberg and Nissan equation: Grunberg and Nissan [51] proposed the following equation for the viscosity measurements in binary mixtures:

$$
\ln \eta=x_{1} \ln \eta_{1}+x_{2} \ln \eta_{2}+x_{1} x_{2} d
$$

where, "d" is Grunberg-Nissan interaction parameter, shows the non-ideality of system by identifying the presence of interactions between the components.

Katti and Chaudhri equation: This equation [52] can be expressed as follows:

$$
\ln \eta V=x_{1} \ln V_{1} \eta_{1}+x_{2} \ln V_{2} \eta_{2}+\frac{x_{1} x_{2} W_{v i s}}{R T}
$$

where, " $\mathrm{W}_{\text {vis }}$ " is Katti and Chaudari interaction parameter.

Similarly Hind equation [53] can be expressed as follows:

$$
\eta=x_{1}^{2} \eta_{1}+x_{2}^{2} \eta_{2}+2 x_{1} x_{2} H_{12}
$$

where, " $\mathrm{H}_{12}$ " is Hind interaction parameter.

FT-IR studies: The hydrogen bonding between the proton donor $\mathrm{N}-\mathrm{H}$ of aniline and carbonyl group $(\mathrm{C}=\mathrm{O})$ of $p$-chloroacetophenone using FTIR spectroscopy is clearly observed. Generally, the intermolecular hydrogen bonding shows sharp peaks, whereas intramolecular hydrogen bonding shows broad peaks. Pure components show only broad bands. The characteristic absorption at 3425 and $3359 \mathrm{~cm}^{-1} \mathrm{~N}-\mathrm{H}$ stretching frequency of aniline. There will be two bands for primary aniline. Only one band for $N$-methylaniline which is observed at $3408 \mathrm{~cm}^{-1}$, while $\mathrm{N}, \mathrm{N}$-dimethylaniline does not show any $\mathrm{N}-\mathrm{H}$ stretching.

\section{Conclusion}

The experimental values of density, viscosity and ultrasonic sound for the binary liquid mixtures of $p$-chloroacetophenone with aniline, $N$-methylaniline and $N, N$-dimethylaniline at four different temperatures $(303.15,308.15,313.15,318.15 \mathrm{~K})$. From these values, excess parameters are calculated and correlated using Redlich-Kister type polynomial equation to derive the coefficients and standard deviation. The observed negative values of $\mathrm{V}^{\mathrm{E}}, \Delta \eta$ and positive values of $\Delta \mathrm{k}_{\mathrm{s}}$ clearly shows that there are strong interactions between the mixing components. The order of interaction among the molecules in binary liquid

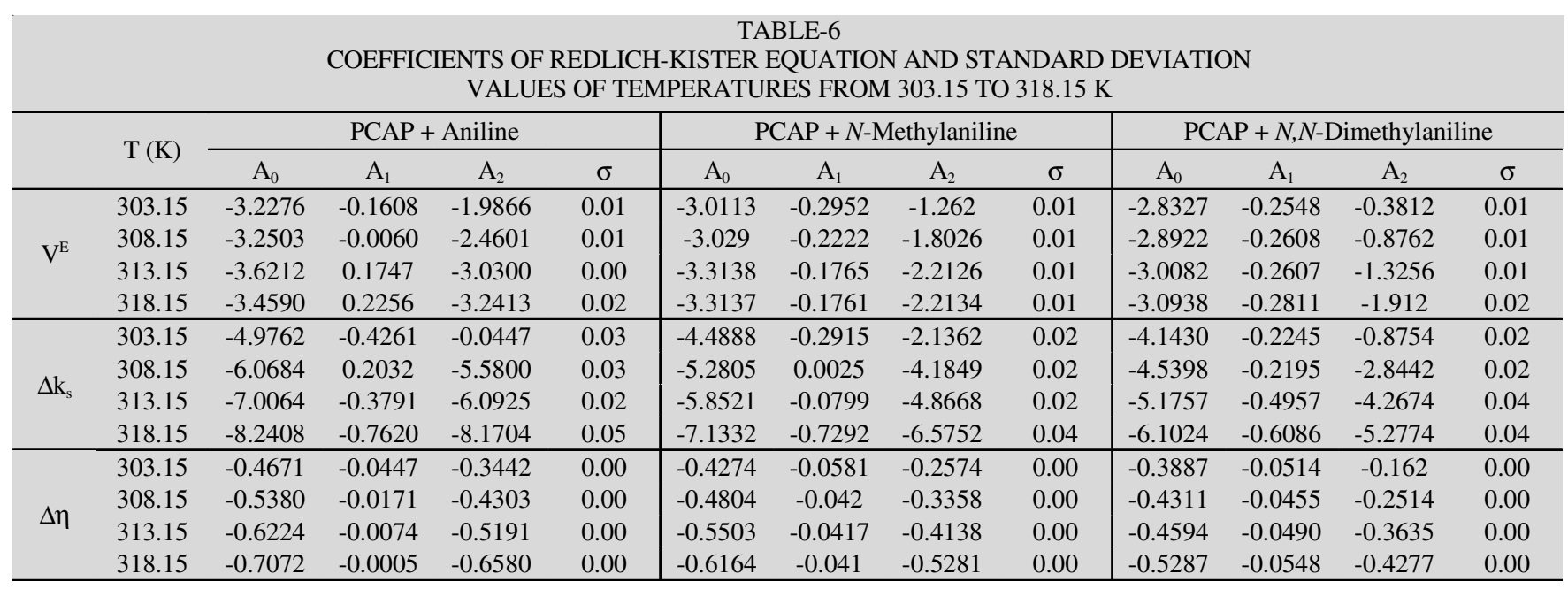


mixtures is as follows: $(\mathrm{PCAP}+\mathrm{AN})>(\mathrm{PCAP}+\mathrm{NMA})>(\mathrm{PCAP}$ + NNDMA). The increasing order of strength with respect to temperature is as follows: $318.15 \mathrm{~K}>313.15 \mathrm{~K}>308.15 \mathrm{~K}>$ $303.15 \mathrm{~K}$.

\section{ACKNOWLEDGEMENTS}

One of the authors, M. Radha Sirija is grateful to University Grants Commission, New Delhi, India for awarding Basic Scientific Research Fellowship in sciences.

\section{CONFLICT OF INTEREST}

The authors declare that there is no conflict of interests regarding the publication of this article.

\section{REFERENCES}

1. A. Díez, J. Largo and J.R. Solana, J. Chem. Phys., 125, 074509 (2006); https://doi.org/10.1063/1.2217944.

2. A. Ali, A.K. Nain, V.K. Sharma and S. Ahmad, Indian J. Phys., 758, 519 (2001).

3. H. Iloukhani, Z. Rostami and N. Afshari, Phys. Chem. Liq., 47, 360 (2009); https://doi.org/10.1080/00319100701824652.

4. M. Gowrisankar, S. Sivarambabu, P. Venkateswarlu and K.S. Kumar, Bull. Korean Chem. Soc., 33, 1686 (2012); https://doi.org/10.5012/bkcs.2012.33.5.1686.

5. R. Balaji, M. Gowri Sankar, M. Chandra Sekhar and M. Chandra Shekar, Phys. Chem. Liq., 54, 422 (2015); https://doi.org/10.1080/00319104.2015.1109996.

6. M. Swetha Sandhya, P. Biswas, N.R. Vinay, K. Sivakumar and R. Dey, J. Mol. Liq., 278, 219 (2019); https://doi.org/10.1016/j.molliq.2019.01.056.

7. D. Rahul, M. Gowri Sankar, M. Chandra Sekhar and D. Ramachandran, J. Therm. Anal. Calorim., 125, 935 (2016); https://doi.org/10.1007/s10973-016-5409-7.

8. P. Nagababu, S. Babu, D.F. Santos and M. Gowrisankar, Chem. Data Collect., 20, 100196 (2019); https://doi.org/10.1016/j.cdc.2019.100196.

9. M. Raveendra, M. Chandrasekhar, K.C. Reddy, A. Venkatesulu, K. Sivakumar and K.D. Reddy, Fluid Phase Equilib., 462, 85 (2018); https://doi.org/10.1016/j.fluid.2018.01.025.

10. A. Shakila, S. Ravikumar, V. Pandiyan and R. Gaba, J. Mol. Liq., 265, 544 (2018); https://doi.org/10.1016/j.molliq.2018.05.130.

11. A.R.M. Khan, M.M.H. Rocky, F.I. Chowdhury, M.S. Ahmed and S. Akhtar, J. Mol. Liq., 277, 681 (2019); https://doi.org/10.1016/j.molliq.2018.12.136.

12. S.J. Kharat and P.S. Nikam, J. Mol. Liq., 131-132, 81 (2007); https://doi.org/10.1016/j.molliq.2006.08.053.

13. B. Nagarjun, A.V. Sarma, G.V. Rama Rao and C. Rambabu, J. Thermodyn., 2013, Article ID 285796 (2013); https://doi.org/10.1155/2013/285796.

14. P. Anila, K.R. Reddy, G. Srinivasa Rao, P.V.S. Sairam, D. Ramachandran and C. Rambabu, J. Chem. Thermodyn., 104, 24 (2017); https://doi.org/10.1016/j.jct.2016.09.010.

15. Partibha, K. Kumar, S. Gahlyan, M. Rani and V. Bhankar, J. Mol. Liq., 259, 167 (2018); https://doi.org/10.1016/j.molliq.2018.03.025.

16. P.V. Rao, T.S. Krishna, M.G. Sankar and K. Ravindhranath, J. Mol. Liq., 222, 873 (2016); https://doi.org/10.1016/j.molliq.2016.07.123.

17. G.P. Dubey, S. Rani and H. Kumar, J. Chem. Thermodyn., 132, 1 (2019); https://doi.org/10.1016/j.jct.2018.12.012.

18. S.M.C. Sousa, E.F.G. Barbosa and I.M.S. Lampreia, J. Chem. Thermodyn., 56, 60 (2013); https://doi.org/10.1016/j.jct.2012.07.012.

19. M. Gowrisankar, P. Venkateswarlu, K. Siva Kumar and S. Sivarambabu, J. Mol. Liq., 173, 172 (2012); https://doi.org/10.1016/j.molliq.2012.06.010.

20. S.J. Tangeda, S. Boodida and S. Nallani, J. Chem. Thermodyn., 38, 1438 (2006); https://doi.org/10.1016/j.jct.2006.01.009.

21. T.S. Jyostna and N. Satyanarayana, Indian J. Chem. Technol., 13, 71 (2006).

22. S.J. Tangeda and S. Nallani, J. Chem. Eng. Data, 50, 89 (2005); https://doi.org/10.1021/je040008e.

23. R. Palepu, J. Oliver and D. Campbell, J. Chem. Eng. Data, 30, 355 (1985); https://doi.org/10.1021/je00041a036.
24. M. Srilatha, D. Chinnarao, G.V. Gaurav and C.V. Padmarao, Chem. Sci. Transac., 5, 223 (2016);

https://doi.org/10.7598/cst2016.1138.

25. I. Alonso, V. Alonso, I. Mozo, I.G. de la Fuente, J.A. González and J.C. Cobos, J. Chem. Eng. Data, 55, 2505 (2010); https://doi.org/10.1021/je900874z.

26. L. Su and H. Wang, J. Chem. Thermodyn., 41, 315 (2009); https://doi.org/10.1016/j.jct.2008.08.013.

27. A.K. Nain, Fluid Phase Equilib., 259, 218 (2007); https://doi.org/10.1016/j.fluid.2007.07.016.

28. M. Gowrisankar, K. Venkateswarlu, S. Sivakumar and S. Sivarambabu, J. Solution Chem., 42, 916 (2013); https://doi.org/10.1007/s10953-013-0003-0.

29. S.L. Oswal, V. Pandiyan, B. Krishnakumar and P. Vasantharani, Thermochim. Acta, 507-508, 27 (2010); https://doi.org/10.1016/j.tca.2010.04.025.

30. G. Korosi and E.S.Z. Kovats, J. Chem. Eng. Data, 26, 323 (1981); https://doi.org/10.1021/je00025a032.

31. V.S. Rao, T.V. Krishna, T.M. Mohan and P.M. Rao, J. Chem. Thermodyn., 100, 165 (2016); https://doi.org/10.1016/j.jct.2016.04.024.

32. M. Aftabuzzaman, M.M. Islam, Nasiruddin, F.R. Rima, M.N. Islam and M.A. Ali, J. Chem. Thermodyn., 96, 181 (2016); https://doi.org/10.1016/j.jct.2015.12.030.

33. I. Alonso, I. Mozo, I.G. de la Fuente, J.A. González and J.C. Cobos, J. Chem. Eng. Data, 55, 5400 (2010); https://doi.org/10.1021/je100472t.

34. S. Kumar and P. Jeevanandham, J. Mol. Liq., 174, 34 (2012); https://doi.org/10.1016/j.molliq.2012.07.025.

35. V. Pandiyan, S.L. Oswal and P. Vasantharani, Thermochim. Acta, 518, 36 (2011); https://doi.org/10.1016/j.tca.2011.02.004

36. V.K. Sharma, S. Solanki, S. Bhagour and D. Sharma, J. Mol. Liq., 188, 258 (2013); https://doi.org/10.1016/j.molliq.2013.09.024.

37. P. Vasundhara, C. Narasimha Rao, L. Venkatramana, K. Sivakumar, P. Venkateswarlu and R.L. Gardas, J. Mol. Liq., 202, 158 (2015); https://doi.org/10.1016/j.molliq.2014.12.024.

38. N.G. Devi, N.V.N.B. Srinivasa Rao, M. Radha Sirija and D. Ramachandran, Korean J. Chem. Eng., 35, 1488 (2018); https://doi.org/10.1007/s11814-018-0053-5.

39. M. Kondaiah, D. Sravana Kumar, K. Sreekanth and D. Krishna Rao, J. Chem. Eng. Data, 57, 352 (2012); https://doi.org/10.1021/je200862b.

40. M. Gowrisankar, P. Venkateswarlu, K. Siva Kumar and S. Sivarambabu, J. Ind. Eng. Chem., 20, 405 (2014); https://doi.org/10.1016/j.jiec.2013.04.035.

41. J.A. Riddick, W.B. Bunger and T.K. Sakano, Organic Solvents Physical Properties and Method of Purifications, Wiley Interscience: New York, vol. 2 (1986).

42. A. Valtz, M. Teodorescu, I. Wichterle and D. Richon, Fluid Phase Equilib., 215, 129 (2004); https://doi.org/10.1016/S0378-3812(03)00364-9.

43. M.H. Kabir, M.A. Motin and M.E. Huque, Phys. Chem. Liq., 42, 279 (2004); https://doi.org/10.1080/0031910042000205346.

44. P. Venkatesu and M.V.P. Rao, J. Chem. Eng. Data, 41, 1059 (1996); https://doi.org/10.1021/je9600919.

45. B. Hawrylak, K. Gracie and R. Palepu, J. Solution Chem., 27, 17 (1998); https://doi.org/10.1023/A:1022636511542.

46. P.K. Pandey, V.K. Pandey, A. Awasthi, A.K. Nain and A. Awasthi, Thermochim. Acta, 586, 58 (2014); https://doi.org/10.1016/j.tca.2014.03.038

47. M.I. Aralaguppi, C.V. Jadar, T.M. Aminabhavi, J.D. Ortego and S.C. Mehrotra, J. Chem. Eng. Data, 42, 301 (1997); https://doi.org/10.1021/je960148r.

48. R.J. Fort and W.R. Moore, Trans. Faraday Soc., 61, 2102 (1965); https://doi.org/10.1039/tf9656102102.

49. R.J. Fort and W.R. Moore, Trans. Faraday Soc., 62, 1112 (1966); https://doi.org/10.1039/tf9666201112.

50. O. Redlich and A.T. Kister, Ind. Eng. Chem., 40, 345 (1948); https://doi.org/10.1021/ie50458a036.

51. L. Grunberg and A.H. Nissan, Nature, 164, 799 (1949); https://doi.org/10.1038/164799b0.

52. P.K. Katti and M.H. Chaudhri, J. Chem. Eng. Data, 9, 442 (1964); https://doi.org/10.1021/je60022a047.

53. R.K. Hind, E. McLaughlin and A. Ubbelohde, Trans. Faraday Soc., 56, 328 (1960); https://doi.org/10.1039/tf9605600328. 